\title{
Hacia una reforma del Estado peruano Pautas para la reestructuración del Poder Ejecutivo ${ }^{1}$
}

\author{
Francisco Sagasti \\ con la colaboración de \\ Gustavo Guerra-García
}

\begin{abstract}
En este trabajo se analiza la necesaria modernización del aparato estatal en el Perú, esspecialmente en cuanto al Poder Ejecutivo, partiendo por un examen de la crisis de gobernabilidad democrática que alli se vive. Haciendo referencia a lo experimentado en diversas regiones del mundo se revela la importancia de la relación entre el Estado, el mercado y la participación de la sociedad civil. Otro factor que se examina es la exigencia de llevar a cabo reformas estructurales e institucionales, luego de las fracasadas políticas de estabilización económicạ. El diagnóstico que el autor elabora de los principales rasgos que presenta el Poder Ejecutivo, especialmente en cuanto al estilo de gestión pública, permite apreciar sus limitaciones e identificar los riesgos que implica no efectuar cambios significativos. Para finalizar, se formulan pautas para desarrollar a largo plazo un proceso de reformas del sistema estatal y del.Poder Ejecutivo peruanos que responda a los desafios del siglo XXI.
\end{abstract}

\section{Introducción.}

Hace poco más de treinta años tuve la oportunidad de realizar mi primer trabajo profesional sobre reforma del Estado. Recién egresado de la Facultad de Ingeniería Industrial de la Universidad Nacional de Ingeniería me integré a un equipo de consultores que estaba reestruc-

\footnotetext{
'Este documento resume los avances de un trabajo que viene realizando AGENDA: Perú sobre reforma del Estado. Se tiene programado avanzar en el diseño organizativo para un Poder Ejecutivo que esté a la altura de los desafíos que, luego de la estabilización económica, sitúe al Perú en el umbral de Siglo XII. Posteriormente se iniciará un amplio proceso de consultas para incorporar nuevos puntos de vista, buscando generar consensos operativos sobre las principales líneas para la reforma del Estado y del Poder Ejecutivo.
} 
turando a la empresa pública más antigua del Perú: la Corporación Nacional de Fertilizantes, fundada hace casi un siglo y medio como la Compañía Administradora del Guano del Perú y mejor conocida como "la guanera". Esta experiencia me permitió trabajar con profesionales de la talla de Luis Rossi Ugarte, ex-Ministro de Agricultura del Presidente Luis Bustamante y Rivero; y también enfrentarme de lleno a la difícil realidad de la administración pública peruana.

No voy a relatar lo mucho que aprendí durante los meses que duró el trabajo. Baste sólo señalar que lo que encontramos en esa empresa poco tenía que ver con los libros de texto que había estudiado en la universidad. Sin embargo, Don Luis nos exhortaba a no considerar "irracionales" o "perversas" las prácticas burocráticas de esa entidad. Por el contrario, nos invitaba a examinar los códigos de comportamiento y la lógica administrativa que había evolucionado durante un siglo, sobreviviendo a una larga serie de gobiernos de la más diversa orientación. Nos advertía también que para romper la inercia burocrática era necesario ser pacientes, tercos y escuchar atentamente lo que nos decían los funcionarios, evitando imponer puntos de vista preconcebidos que generalmente pecaban de simplistas.

Mi experiencia con muchas otras entidades públicas, organismos internacionales y empresas privadas ha confirmado lo que aprendí de Don Luis Rossi Ugarte ( $\mathrm{y}$ de maestros como Eric Trist, Rusell Ackoff, Stafford Beer y Yehezkel Dror). Es necesario cuidarse de la ilusión tecnocrática de que existen soluciones mágicas -sịmples, lógicas, rápidas y eficientes- para la modernización de la administración pública. Cuando se trata de reformar instituciones, los avances parciales pero sólidos, capaces de obtener un amplio apoyo que haga dificil su reversión, son preferibles a los grandes saltos hacia adelante que rara vez llegan a consolidarse y sobrevivir a quienes los impulsan. Estas advertencias cobran actualidad con el intento que viene realizando el gobierno para modernizar el aparato estatal.

Una apreciación de la crisis de gobernabilidad democrática que experimenta nuestro país desde hace algunos años es un buen lugar para iniciar este documento sobre reforma del Estado, y también para contrarrestar las ilusiones tecnocráticas, enemigas de las reformas duraderas. A continuación resumiremos apretadamente las lecciones de la experiencia y de la teoría acerca del papel del Estado, del sector privado y de las organizaciones de la sociedad civil en el proceso de desarrollo. Luego presentaremos algunos comentarios sobre la relación entre los procesos de estabilización y ajuste estructural por un lado, y la reforma del Poder 
Ejecutivo por otro. Las dos últimas secciones ofrecen un diagnóstico de los principales problemas del Ejecutivo en el Perú y proponen: pautas para iniciar un proceso de reformas de largo alcance.

\section{El contexto de la reforma del Estado.}

Tres conjuntos de factores deben șer tomados en cuenta al examinar el contexto que enmarca: Ios esfuerzos para reformar el Estado peruano: la crisis de gobernabilidad democrática que hemos experimentado durante el último decenio y medio; los avances en el contexto internacional acerca de las relaciones entre el Estado, el sector privado y las organizaciones de la sociedad civil; y las exigencias de la transición de un período de estabilización económica a uno de reformas estructurales e institucionales para promover el desarrollo humano.

\section{Gobernabilidad democrática en el Perú.}

El primer informe de Agenda: Perú, Democracia y Buen Gobierno; resumió los problemas de gobernabilidad democrática en nuestró país ên los siguientes términos:

El Perú está atravesando por un proceso de cambio social y profundo y acelerado, gestado durante varios siglos. Estos cambios corresponden, en cierta medida, a las transformaciones que tienen lugar en el mundo, pero el carácter multicultural, heterogéneo-y complejo de la sociedad peruana los hace más agudos y los amṕlifica. En la transición a un nuevo siglo y a un nuevo milenio vivimos en un tiempo de enormes desafios y: oportunidades para forjar nuestro propio destino comonación.

Durante los decenios de 1960 a 1980, esțos procesos de cambio generaron una serie de demandas de bienes, servicios, orden y paz que desbordaron la capacidad de las instituciones del Fstado, del sector productivo y de las organizaciones sociales para satisfacerlas. Estas demandas se derivan del crecimiento de la poblacion y deil hecho de que los sectơres marginados ya no están dispuestos a seguir tolerando las injusticias que caracterizaron ai Perú por siglos. El desfase entre las demandas y la capacidad para satisfacerlas llevó al cuestionamiento géneralizado de las instituciones políticas, económicas y sociales, y generó ună crisis de gobernabilidad. Durante el decenio de 1980 coincidieron el surgimiento y propagación de la violericia terrorista de Sendero Luminoso, el desmanejo económico que llevó a la hiperinflación hacia fines del decenio, y la presencia de gobiernos democráticos. Esto hizo que la crisis de gobernabilidad adquiriera la particular naturaleza de una crisis de gobernabilidad democrática. 
El avance hacia un Perú que cuente con una sociedad civil organizada y activa, una economía moderna y competitiva, y un Estado representativo y eficiente se dará por la confluencia e interacción de tres procesos: la democratización socialœ que propicia una mayor igualdad y requiere de la creación de mayores oportunidades para todos los peruanos; la modernización productiva, que está extendiendo el ámbito en el cual operan los mercados y busca elevar la productividad de las empresas peruanas; y la legitimación de las instituciones estatales, que apunta hacia una reforma integral del Estado para hacerlo más representativo y eficiente. Estos procesos deben estar orientados por una visión compartida del futuro del Perú, capaz de movilizar esfuerzos y voluntades. ${ }^{2}$

El imperativo de legitimar a las instituciones del Estado es una de las principales conclusiones a que llegamos en el amplio proceso de consultas que realizamos en Agenda: Perú. El proceso de cambios acelerados y profundos que estamos viviendo exige una adecuación de las instituciones estatales a las nuevas realidades del pais y del contexto internacional. Además de la reforma de los poderes del Estado-Ejecutivo, Legislativo y Judicial- esto requiere de un profundo examen del régimen político vigente. Asuntos tales como el peso relativo de los poderes del Estado y las relaciones entre ellos deben ser debatidos ampliamente en todas las instancias de la vida política nacional y decididos por consenso, sin imposiciones ni actitudes prepotentes.

La reforma del Poder Ejecutivo merece especial atención en este proceso de adecuación de las instituciones del Estado. Es necesario replantear las funciones, estructura, organización y tamaño del Poder Ejecutivo, particularmente en vista del nuevo papel que las reformas económicas asignan al Estado y de la experiencia del colapso de las instituciones públicas en el decenio de los ochenta. La nueva división del trabajo que está surgiendo entre los sectores público, privado y de la sociedad civil, hace que las empresas privadas y las organizaciones de la sociedad civil estén asumiendo funciones que antes eran responsabilidad del Estado. Un ejemplo claro está dado por la provisión de servicios sociales básicos, de infraestructura y aun de seguridad.

La reforma del Ejecutivo requiere de una revisión de la estructura de los ministerios en el gobierno central, la creación y el fortalecimiento de instancias de regulación de la actividad privada (sobre todo en el caso de los servicios públicos privatizados), la creación de un sistema nacional

\footnotetext{
${ }^{2}$ Francisco Sagasti, Pepi Patrón, Nicolás Lynch y Max Hernández, Democracia y Buen Gobierno, (Lima: Apoyo/AGENDA:Perü), 2a edición, 1996, pp. 129-130.
} 
para definir prioridades de inversión pública, la modernización y la flexibilización de lós sistemas de control para evitar la corrupción y el despilfarro. de :recursos, el establecimiento de nuevos sistemas de planeamiento yy control presupuestal, la definición de nuevas formas de vinculación entre el gobierno central y los gobiernos locales, y la creación de un sistema de servicio civil para los funcionarios públicos. Además, el énfasis que debe otorgar el Poder Ejecutivo a la provisión de servicios sociales básicos plantea nuevas exigencias de carácter administrativo, y también la necesidad de establecer vínculos operativos con las organizaciones de la sociedad civil y del sector privado. Todo esto indica la urgencia de diseñar nuevas estructuras administrativas y nuevos procedimientos para la actuación del Poder Ejecutivo, y para su interacción con otras entidades públicas, privadas y no gubernamentales.

\section{La experiencia internacional.}

Durante los últimos cuatro decenios hemos aprendido, tanto en América Latina como en otras regiones del mundo en desarrollo, que las sociedades que más han progresado han sido aquellas que lograron éstablecer relaciones de complementariedad entre el Estado, el libre juego de las fuerzas de mercado y la participación activa de la sociedad civil. ${ }^{3}$

Esto implica evitar convertirnos, una vez más, en prisioneros de una de las tres utopías que, llevadas a sus extremos, han causado mucho daño en América Latina. Me refiero a la utopía estatista, en la cual el Estado puede y debe hacerlo todo; la utopía de mercado, en la cual basta sólo con dejar que funcionen las fuerzas de mercado para resolver todos nuestros problemas; y la utopía basista, en la cual la acción de las bases organizadas es lo único que puede llevarnos hacia el desarrollo.

Si bien ninguna de estas utopías ha denominado completamente el escenario político a lo largo del tiempo, no es menos cierto que en determinados momentos alguna de ellas ha llevado la voz cantante. Por ejemplo, durante el último cuarto de siglo, en el Perú hemos experimentado el predominio de la utopía estatista a fines del decenio de los sesenta y principios del de los setenta, la pugna entre la utopía basista y la visión estatista durante el gobierno entre 1985 y 1990 , y el predominio de la utopía de mercado durante los últimos seis años.

Para avanzar en el diseño y puesta en práctica de estrategias de desarrollo que sean viables y sostenibles en la transición a un nuevo siglo,

\footnotetext{
${ }^{3}$ Banco Mundial, La Tarea Acuciante del Desarrollo-Informe Sobre el Desarrollo Mundial 1991, Washington D.C., 1991.
} 
es necesario evitar los extremos que representan estas tres utopías. En contraposición; deberíamos buscar lo que podríamos llamar la utopía del justo medio, caracterizada por el equilibrio entre la acción del Estado, de las fuerzas de mercado y de la enorme variedad de organizaciones de la sociedad civil. Sólo un balance adecuado entre estos tres protagonistas de nuestro desarrollo hará que el Perú entre con buen pie al siglo XXI.

Los mercados competitivos pueden asignar los recursos eficientemente tanto en los procesos de producción como de distribución de bienes y servicios pero, para que ello ocurra, los diferentes mercados requieren de un marco legal y regulatorio operativo que sólo los gobiernos pueden suministrar, así como de la vigilancia y el apoyo social que sólo las organizaciones de la sociedad civil pueden proporcionar. Además, en determinadas circunstancias existen fallas de mercado (externalidades, bienes públicos, información incompleta) que justifican una intervención del gobierno para incentivar o simular la competencia. De aquí surge la aparente paradoja de que para que el mercado funcione se requiere de la intervención estatal y de la activa participación de la sociedad civil.

Por otra parte, la experiencia internacional indica que la descentralización, la privatización de servicios públicos, el fortalecimiento de la gestión pública superior y la transparencia en el ejercicio de las atribuciones del gobierno han coadyuvado al mejor desempeño económico y social. La descentralización de las decisiones gubernamentales y de la provisión de servicios sociales de todo tipo, así como la consolidación de gobiernos regionales y locales, permite una mejor respuesta a las demandas locales, sobre todo en países heterogéneos. Además, permite resolver las asimetrías de información que produce una estructura de decisiones excesivamente concentrada y facilita un mejor control de la función pública por parte de la ciudadanía.

La búsqueda de una relación más eficiente entre los sectores público y privado ha generado programas de privatización que redujeron significativamente las actividades empresariales del Estado y transformaron en privadas a muchas actividades del sector público. Los programas más exitosos de transferencia de responsabilidades de servicios públicos al sector privado también han propiciado una mayor participación de las organizaciones de la sociedad civil. Por ejemplo, el Banco Mundial ha señalado algunos factores asociados a las privatizaciones exitosas en infraestructura, tales como promover la competencia en la provisión de los servicios, brindar a los usuarios canales de opinión para evaluar la calidad de los servicios, y asociar a los sectores público, privado y a las 
organizaciones de la sociedad civil en el financiamiento de los proyectos y la operación de los servicios.

Las innovaciones en gestión pública han puesto énfasis en el fortalecimiento de la gestión pública superior y en el estudio de las condiciones institucionales que afectan el desempeño de las agencias gubernamentales. ${ }^{5} \mathrm{La}$ gestión pública superior se refiere a las funciones y actividades que realizan las autoridades políticas-administrativas al más alto nivel, e incluye instancias tales como la oficina del Primer Ministro, los ministros de Estado, los vice-ministros o secretarios de Estado, los directivos de comisiones de alto nivel y los presidentes de agencias especializadas, todas ellas con sus respectivos gabinetes de asesores.

Los avances en la gestión pública superior están asociados con mejoras en planeamiento estratégico, el uso de sistemas de información, la creación de procedimientos de coordinación, los programas de reclutamiento y formación de personal clave, el establecimiento de objetivos de política, la definición de prioridades para la acción gubernamental, y el seguimiento y evaluación de los resultados de las políticas y programas. Fortalecer la gestión pública superior implica contar con decenas y aun centenares de profesionales altamente capacitados y con experiencia en el sector público, algo que toma tiempo y requiere, a su vez, de una concepción estratégica de mediano y largo plazo.

Por último, los enfoques recientes sọbre la gobernabilidad y el desarrollo han puesto énfasis en las condiciones necesarias para un manejo más efectivo y eficiente del aparato del Estado. La transparencia en la gestión pública, los procedimientos y prácticas para una mejor rendición de cuentas (accountability) y la existencia de marcos legales estables se consideran elementos claves para mejorar el desempeño del sector público. ${ }^{6}$ Entre otras cosas, esto implica otorgar una mayor autonomía económica, financiera, administrativa y funcional a las diversas entidades estatales, estableciendo criterios de evaluación por anticipado y mejorando los sistemas de manejo y control presupuestario. La idea central apunta hacia cambiar estos sistemas de modo que los líderes de

\footnotetext{
${ }^{4}$ Banco Mundial, Infraestructura y Desarrollo: Informe sobre el Desarrollo Mundial 1994, Washington, D.C., 1994.

${ }^{5}$ Véase Enrique Correa Elias, "Perspectivas de la Modernización del Estado y las Políticas Públicas en América Latina", trabajo presentado en el taller sobrc "Estado, interdependencia y soberania", UNESCO, Cartagena de Indias, julio de 1995.

${ }^{6}$ Banco Mundial, Gobernabilidad y Desarrollo, (Lima: INIDEN/IDEl, Universidad Católica del Perú, 1996).
} 
cada organización dediquen más tiempo y atención a los resultados que: a los procedimientos.

\section{De la estabilización a las reformas estructurales.}

Los excesos de la "década perdida" en América Latina, que en países como el Perú llevaron a procesos hiperinflacionarios, hicieron necesāriăs" políticas de estabilización para reestablecer los equilibrios macroeconómicos alterados, en muchos casos, por la excesiva intervención estatal. A medida que se fue recuperando la estabilidad macroeconómica y se hizo necesario avanzar en las reformas de política económica y social, se tomó conciencia de los diferentes requerimientos institucionales de fases de estabilización y reformas estructurales.

Moisés Naím ha llamado la atención acerca de las diferencias que existen entre las fases de estabilización y de reformas estructurales en lo referente a prioridades, estrategias, instrumentos y metas, así como en to que concierne a los actores, los impactos, la complejidad y la naturaleza de los costos políticos. ${ }^{7}$ Los cuadros $\mathrm{N}^{\circ} 1$ y $\mathrm{N}^{\circ} 2$ resumen sus planteamientos, y hacen ver muy claramente que los estilos de gobierno apropiados para la fase de estabilización son totalmente inadecuados para la fase de reformas estructurales. Esto señala muy claramente que no eś posible avanzar en la reforma del Estado sin profundizar y consólidar las instituciones asociadas con la gobernabilidad democrática.

\section{Crisis del Estado y ajuste estructural en el Perú.}

Los desequilibrios macroeconómicos del decenio de los ochenta-cuyas expresiones más visibles fueron la crisis de la deuda, la recesión económica y las altas tasas de inflación-motivaron el diseño y puesta en práctica de programas de estabilización y ajuste estructural en casi toda América Latina. El Perú fue una excepción: hacia fines de la "década perdida" el gobierno continuaba experimentando con políticas estatistas y heterodoxas que desembocaron en la hiperinflación más larga de nuestra historia. Esto agudizó la crisis de gobernabilidad democrática, el deterioro social y devaluó las instituciones, sobre todo en el sector público. También hizo necesario un drástico programa de estabilización y ajuste que agudizó los problemas sociales a principios del decenio de los noventa.

\footnotetext{
${ }^{7}$ Moisés Naim, "Latin Americas Journey to the Market: from Macroeconomic Shocks to Institutional Therapy", Washington, D.C., Carnegie Endowment for International Peace, 1994.
} 
Deterioro social y crisis institucional.

Mientras que otros países buscaban un mejor equilibrio entre la acción del Estado y las fuerzas del mercado, el gobierno de Alan García insistió en políticas estatistas y populistas que hicieron un enorme daño a la economía nacional. Entre 1985 y 1990 las empresas estatales perdieron US $\$ 1.650$ millones, la presión tributaria cayó de $17,3 \%$ a sólo $4,9 \%$ del PBI, los depósitos del sistema financiero se redujeron de $23 \%$ a $5 \%$ del PBI y el Perú dejó de ser sujeto de crédito para la banca privada internacional y de los organismos multilaterales. ${ }^{8}$ Los empresarios locales y extranjeros perdieron la confianza en la economía peruana, trasladaron sus inversiones a otros países, y en vez de esforzarse por mejorar la eficiencia de sus empresas se dedicaron a la especulación financiera para mantener el valor de sus activos.

Hacia fines del decenio de 1980, el PBI real per capita se redujo a un nivel inferior al de:1960, Como reflejo de este deterioro se produjo la degradación tanto de la función pública como de los servicios públicos. Los gastos en educación y salud se redujeron drásticamente, y la demanda insatisfecha por estos servicios aumentó al mismo tiempo que se redujo en forma alarmante la calidad de estos servicios. Por ejemplo, en Lima Metropolitana -en donde se concentra un tercio de la población nacional-el $32 \%$ de los habitantes carecía de conexión de agua potable y el resto sufría algún tipo de racionamiento; y el gasto real promedio per capita de los habitantes de Lima Metropolitana se redujo en un 52,7\% entre 1985 y 1990 .

Por otra parte, el gasto en mantenimiento vial se redujo a sólo el $14 \%$ de lo requerido, por lo que sólo el $20 \%$ de la red vial se mantuvo en buen estado. La capacidad operativa de las empresas eléctricas se redujo en $30 \%$, mientras que el déficit energético aumentó significativamente, lo que obligó a muchas empresas a instalar su propia capacidad de generación a un costo muy alto. Los puertos peruanos se encontraban entre los más caros del mundo y el Perú batió el récord de demanda insatisfecha por servicio telefónico, medida como porcentaje de personas en lista de espera $(62 \%)$ respecto de la demanda registrada (líneas disponibles más líneas solicitadas).

\footnotetext{
${ }^{8}$ Las cifras de esta sección provienen de varios números de la revista Perú Económico entre 1990 y 1996, publicado por APOYO S.A.

${ }^{9}$ Paul Glewwe y Gullete Hall, "Poverty and Inequality during Unorthodox Adjustment: the Case of Peru 1985-1990", Washington D.C., Banco Mundial, 1992.
} 
La policía y el Poder Judicial también experimentaron un deterioro significativo. En un contexto de crisis económica y de violencia terrorista, los índices de criminalidad se elevaron considerablemente $y$ a finales del decenio de los ochenta, 6,4 personas morían cada día como consecuencia de la violencia urbana no política. Los jueces fueron amenazados y amedrentados por terroristas, narcotraficantes y delincuentes. La institución policial sè deterioró por efecto de la violencia terrorista (que le costó la vida a 1.300 policías entre 1980 y 1990), y de la corrupción interna:que generó desconfianza y temor de la población frente a la policía. Los establecimientos penales también fueron afectados por la irresponsabilidad en el manejo de los asuntos públicos. Entre 1985 y 1990, aumentó drásticamente el número de inculpados sin sentencia, se sobrepoblaron las cárceles, se reforzaron las mafias en los penales y creció el control de pabellones enteros por parte de grupos terroristas.

Estos problemas llevaron a un virtual colapso del Estado peruano. En líneas generales, como señaló Richard Webb, desde 1975 el sector público se encontraba en un franco proceso de desintegración y se produjo una privatización de facto, que no obedeció a programa político alguno. ${ }^{10}$ Entre 1975 y 1990 la participación de los gastos y los ingresos del gobierno en el PBI se redujo considerablemente, el valor de las ventas de las empresas públicas cayó de $27,5 \%$ a $9,5 \%$, se debilitó significativamente la capacidad reguladora del Estado con el consecuente aumento de las actividades informales y se perdió el control político de una cuarta parte del deterioro nacional. De esta manera, en el Perú tuvo lugar un proceso de "privatización no deseada y desordenada en actividades como los servicios de correo y transporte, educación, salud, suministro de agua potable, generación de energía, y hasta la seguridad pública y la administración de la justicia"."

Cabe destacar que, en algunos casos, los procesos de privatización resolvieron situaciones de emergencia. Por ejemplo, las rondas vecinales de patrullaje llegaron a agrupar a un cuarto de millón de campesinos en 3.000 comités de autodefensa y tuvieron un papel decisivo en la derrota de Sendero Luminoso. En forma similar, el suministro de agua potable por parte de camiones cisterna privados resolvió el problema de abastecimiento de este bien esencial, si bien a un precio muy superior al de Sedapal. Sin embargo, otras privatizaciones de facto fueron realmente

\footnotetext{
${ }^{10}$ Prólogo al libro de Carlos Paredes y Jeffrey Sachs, Estabilización y Crecimiento enel Peni, (Lima: Grade, 1991).

${ }^{11}$ Véase: "Nuevas Ideas para Viejos Problemas", Perú Económico, Vol. XVII, No8, agosto 1994.
} 
indeseables, tales como el tráfico de líneas telefónicas que elevó el valor de la línea a niveles inalcanzables por la gran mayoría de peruanos, y los ajusticiamientos populares en los sectores a los cuales los sistemas de seguridad y administración de justicia no funcionaban o tardaban mucho en llegar.

El menosprecio a la legalidad formal, el descrédito de las instituciones públicas, y la arbitrariedad y el abuso de poder se convirtieron en rasgos distintivos del ejercicio del poder y de la autoridad política a fines del decenio de los ochenta y principios de los noventa. En prácticamente todos los ámbitos de la vida nacional esto llevó a un quiebre de las condiciones mínimas necesarias para establecer y mantener reglas de juego estables, definidas de común acuerdo y respetadas por todos. En contraste con la situación imperante en el Perú, los avances recientes en el campo de la economía institucional ${ }^{12}$ señalan con claridad que $\sin$ instituciones estables que generen incentivos para el mejor desempeño de todo tipo de organizaciones, el desarrollo económico y social es algo prácticamente imposible.

\section{Estabilización económica y reforma del Estado.}

En situaciones de hiperinflación como la que afectó al Perú en 1990 la estabilización macroeconómica se transforma en algo absolutamente prioritario. Las observaciones de Naím (véase cuadros $\mathrm{N}^{\circ} 1$ y $\mathrm{N}^{\circ} 2$ ) sobre las características institucionales del proceso de estabilización sugieren que se requiere de gran determinación política y de un gobierno enérgico para estabilizar una economía en forma exitosa.

Sin embargo, una vez lograda la estabilidad macroeconómica en el corto plazo, es necesario proceder con reformas estructurales, y en particular con una reforma de las instituciones del Estado adoptando una perspectiva de mediano y largo plazo. Pese a los intentos del gobierno, esto no ha sucedido durante los últimos años. A la luz de las contribuciones de varios autores que han estudiado la economía política de la estabilización y el ajuste estructural (Przaworski, Haggard, Kaufman, Naím, Nelson), es posible apreciar los condicionantes políticos e institucionales que en el caso peruano han impedido la transición ordenada de la fase de estabilización a la de reformas estructurales e institucionales.

Entre los factores determinantes del curso de los programas de estabilización y reformas estructurales es posible identificar:

\footnotetext{
${ }^{12}$ North, Douglas, Institutions, Institutional Change and Economic Performance, (Cambridge University Press, 1990).
} 


\section{Cuadro $\mathrm{N}^{0} 1$}

\section{De la estabilización a las reformas institucionales: prioridades, estrategias e instrumentos}

\begin{tabular}{|c|c|c|}
\hline Aspectos/Etapas & Estabilización & $\begin{array}{c}\text { Reformas } \\
\text { institucionales }\end{array}$ \\
\hline Prioridades & $\begin{array}{l}\text { - Reducir la inflación. } \\
\text { Reestablecer el cre- } \\
\text { cimiento. }\end{array}$ & $\begin{array}{l}\text { - Mejorar las condiciones sociales. } \\
\text { - Mejorar la competitividad } \\
\text { internacional. } \\
\text { - Mantener la estabilidad } \\
\text { macroeconómica. }\end{array}$ \\
\hline $\begin{array}{l}\text { Estrategias de la } \\
\text { reforma }\end{array}$ & $\begin{array}{l}\text { Modificar las regias de } \\
\text { juego macroeconómi- } \\
\text { cas. } \\
\text { - Reducir el tamaño y } \\
\text { ámbito del Estado. } \\
\text { - Desmantelar las insti- } \\
\text { tuciones del protec- } \\
\text { cionismo y del } \\
\text { estatismo. }\end{array}$ & $\begin{array}{l}\text { Creación y rehabilitación de } \\
\text { instituciones. } \\
\text { Elevar la competitividad del } \\
\text { sector privado. } \\
\text { - Reformar la provisión y } \\
\text { financiación de servicios sociales. } \\
\text { - Creación de las instituciones } \\
\text { económicas del capitalismo. } \\
\text { Nueva inserción económica } \\
\text { internacional. }\end{array}$ \\
\hline $\begin{array}{l}\text { Instrumentos y } \\
\text { metas típicos }\end{array}$ & $\begin{array}{l}\text { - Recortes presu- } \\
\text { puestarios. } \\
\text { - Liberalización de pre- } \\
\text { cios. } \\
\text { - Liberalización del } \\
\text { comercio internacional } \\
\text { y la inversión extran- } \\
\text { jera. } \\
\text { - Desregulación del sec- } \\
\text { tor privado. } \\
\text { - Privatizaciones fáciles. } \\
\text { - Creación de fondos so- } \\
\text { ciales de emergencia } \\
\text { al margen de los Mi- } \\
\text { nisterios. }\end{array}$ & $\begin{array}{l}\text { - Reforma de la administración } \\
\text { pública. } \\
\text { - Reorganización del gobiemo } \\
\text { central. } \\
\text { - Modernización de la } \\
\text { adninistración de justicia, } \\
\text { - Potenciar la capacidad reguladora } \\
\text { del Estado (monopolios naturales, } \\
\text { servicios públicos, medio } \\
\text { ambiente, sector financiero). } \\
\text { Reconyersión sectorial. } \\
\text { - Privatizaciones complejas } \\
\text { (concesiones de obras e } \\
\text { infraestructura de servicios } \\
\text { públicos). } \\
\text { Promover las exportaciones. } \\
\text { - Reestructurar relaciones entre el } \\
\text { gobierno central y los gobiernos } \\
\text { subnacionales. }\end{array}$ \\
\hline
\end{tabular}




\section{Cuadro $\mathrm{N}^{0} 2$}

\section{De la estabilización a las reformas institucionales: actores, impacto, complejidad y costos políticos}

\begin{tabular}{|c|c|c|}
\hline Aspecto/Etapas & Estabilización & $\begin{array}{c}\text { Reformas } \\
\text { institucionales }\end{array}$ \\
\hline Actores principales & $\begin{array}{l}\text { - Presidente de la } \\
\text { República. } \\
\text { - Gabinete económico. } \\
\text { - Banco Central. } \\
\text { - Organismos multilater- } \\
\text { ales (FMI, Banco } \\
\text { Mundial y BID). } \\
\text { - Grupos financieros pri- } \\
\text { vados e inversionistas } \\
\text { extranjeros. }\end{array}$ & $\begin{array}{l}\text { - Presidencia y Consejo de } \\
\text { Ministros. } \\
\text { - Congreso. } \\
\text { - Burocracia. } \\
\text { - El Poder Judicial } \\
\text { - Sindicatos y sector } \\
\text { social-voluntario. } \\
\text { - Partidos políticos. } \\
\text { - Medios de comunicación. } \\
\text { - Gobiemos subnacionales. } \\
\text { - Sector privado. }\end{array}$ \\
\hline $\begin{array}{l}\text { Impactos de las refor- } \\
\text { mas }\end{array}$ & $\begin{array}{l}\text { - Inmediata. } \\
\text { - Perfil alto del sector } \\
\text { público. }\end{array}$ & $\begin{array}{l}\text { Mediano y larzo plazo. } \\
\text { - Perfil bajo del sector público. }\end{array}$ \\
\hline $\begin{array}{l}\text { Complejidad técnica } \\
\text { y administrativa de } \\
\text { las reformas }\end{array}$ & - Baja y moderada. & - Muy alta. \\
\hline $\begin{array}{l}\text { Naturaleza de los cos- } \\
\text { tos politicos }\end{array}$ & $\begin{array}{l}\text { Costos de las correc- } \\
\text { ciones de corto plazo } \\
\text { distribuidos entre la } \\
\text { mayoría de la } \\
\text { población. }\end{array}$ & $\begin{array}{l}\text { Eliminación permanente de } \\
\text { privilegios a determinados grupos } \\
\text { y barreras a la competencia. }\end{array}$ \\
\hline $\begin{array}{l}\text { Principal desafio del } \\
\text { gobierno. }\end{array}$ & $\begin{array}{l}\text { - Gestión macroe- } \\
\text { conómica a través de } \\
\text { ełites aisladas. }\end{array}$ & $\begin{array}{l}\text { - Gestión participativa del cambio } \\
\text { institucional. } \\
\text { - Logro de consensos, movilización } \\
\text { de la opinión pública y } \\
\text { concertación. } \\
\text { - Desarrollo institucional muy } \\
\text { dependiente de tecnócratas del } \\
\text { sector público. }\end{array}$ \\
\hline
\end{tabular}


La percepción de la crisis económica y política. A fines del decenio de los noventa, el Perú llegó a una situación de angustia generalizada por la violencia terrorista y la hiperinflación. Ante la ciudadanía esto justificó medidas drásticas y radicales, aplicadas ér un contexto de emergencia nacional. Sin embargo, superada la situación de emergencia cambia la naturaleza de los problemas y la percepción ciudadana sobre las prioridades nacionales. Mantener el estilo de gobierno que fue útil para superar la emergencia puede ser contraproducente cuando se trata de resolver los problemas del desarrollo económico y social en el mediano y largo plazo.

La escasa capacidad técnica y administrativa del gobierno. La reducción de las remuneraciones en el sector público y el desprestigio generalizado de las instituciones gubernamentales durante el decenio de los ochenta, generaron una deserción en masa de los mejores profesionales del sector públicô quienes se trasladaron hacia el sector privado:y hacia el extranjero. Como consecuencia, se produjo una reducción drástica de la capacidad operativa de los ministerios, las superintendencias, las empresas públicas y las instituciones descentralizadas. Además; los programas de incentivos para promover renuncias hicieron que los mejores profesionales dejaran el sector público.

El programa de estabilización y ajuste se limitó a fortalecer unas pocas entidades públicas, tales como la SUNAT y la SUNAD, que se convirtieron en islas de modernidad administrativa en un mar de organizaciones estatales burocratizadas y sin capacidad de dirección. Como resultado, en la mayoría de las instituciones públicas se cerró la posibilidad de diseñar y poner en práctica reformas, $y$ de generar planes y programas sectoriales de largo plazo. Pese al tiempo transcurrido desde el inicio del programa de estabilización y ajuste, la mayoría de los ministerios no ha acumulado aún la masa crítica de personal calificado con conocimientos y experiencia, y la información necesaria para formular estrategias y políticas de mediano y largo plazo: $\cdots: \cdots$

Estilo de gobierno y liderazgo político. EI Presidente Alberto Fujimori llegó al poder sin partido político, sin programa de gobierno y sin bases de apoyo organizadas. Desde el inicio de su gestión privilegió su-relación con los organismos multilaterales y con las Fuerzas Armadas. El FMI, el Banco Mundial y el BID ofrecieron propuestas de políticas consistentes que permitieron subsanar la inexistencia de planes de gobierno y avanzar con el proceso de la reinserción 
económica, mientras, que las Fuerzas Armadas se encargaron de la lucha antisubversiva y le dieron una base de apoyo político interno. La coyuntura política al inicio del decenio de los noventa determinó que no se debatieran ni se plantearan alternativas al programa de estabilización y reformas económicas del gobierno, excepto por algunos sectores de oposición radical. No hubo incentivo alguno para que el gobierno estableciera canales de comunicación y diálogo con los diferentes grupos de ciudadanos. Sin embargo, superada la situación de emérgencia económica y política, el estilo de gobierno se mantuvo sin modificaciones.

Las principales decisiones de gobierno continúan tomándose en función de las percepciones del Presidente, quien parece estar en campaña política permanente, así como las de un grupo muy reducido de asesores y funcionarios que realiza consultas sólo de manera esporádica y selectiva, y que no rinde cuentas. Como resultado, se ha acentuado la tendencia a formular propuestas de política de manera poco transparente y con criterios de corto plazo.

A estos factores, que ayudan a comprender los problemas que enfrenta el Perú en la transición hacia un manejo más institucionalizado de las reformas de política económica y social, se une la escasa influencia política de la oposición. Como consecuencia, al interior del gobierno, sobre todo en el Poder Ejecutivo y en el Congreso, no existen condiciones e incentivos para el diseño y puesta en práctica de estrategias y políticas de largo plazo, entre las cuales se encuentra la reforma del Estado peruano. Por el contrario, la forma en que se ejerce el poder político lleva a una concentración de decisiones en pocas manos, socava los esfuerzos para reformar las instituciones públicas y debilitá los procesos de planeamiento estratégico y descentralización. Si esto no se modifica significativamente no será posible avanzar hacia una reforma del Estado duradera, coherente y capaz dè sustentar un proceso de desarrollo económico y social en la transición hacia el siglo XXI.

Poder Ejecutivo: limitaciones actuales y riesgos de "más de lo mismo".

Las apreciaciones acerca del contexto de la reforma del Estado en el Perú nos permiten ubicar los planteamientos acerca de la modernización del Poder Ejecutivo en su dimensión adecuada. Partiendo de una caracterización de la forma en que el estilo de gobierno se manifiesta en el Poder Ejecutivo, es posible identificar los riesgos de continuar con "más de lo mismo" durante los próximos años. 


\section{Limitaciones generales y específicas de la gestión pública.}

Los principales problemas de carácter general de la gestión pública del Poder Ejecutivo pueden resumirse de la siguiente manera;

- Ausencia de una estrategia de desarrollo integral y coherente, y predominio de criterios "pragmáticos" de corto plazo.

- Falta de transparencia, escaso debate público ${ }_{3}$ procedimientos de consulta selectivos y cerrados, y poco interés en la formulación de consensos, aun en aquellos temas de claro interés nacional.

- La política macroeconómica se ha convertido en el principal, sino en el único, determinante del conjunto de políticas en todos los sectores de la actividad gubernamental. Consideraciones referentes al mantenimiento de los equilibrios : macroeconómicos -austeridad fiscal, políticas de endeudamiento público, reducción del número de funcionarios gubernamentales, asignación de recursos para el pago de la deuda externa, entre otros- condicionan fuertemente el diseño y la ejecución de políticas sectoriales.

- La reestructuración administrativa del Poder Ejecutivo está desarticulada de los objetivos de política de cada uno de los sectores, agencias y entidades que la conforman, y está fuertemente condicionada por los procesos de privatización. Existe poca coordinación intersectorial y se carece de un sistema de planeámiento estratégico que asegure la coherencia del conjunto de políticas gubernamentales.

- La mayoría de las políticas en los ámbitos de la macroeconomía y de los diferentes sectores se formulan a escala nacional, sin tomar en cuenta la diversidad de situaciones locales Se centralizan decisiones que deberían tomarse en los niveles regional y local. La mayor parte del presupuesto nacional to manejan el Ministerio de Economía y Finanzas y el Ministerio de la Presidencia.

- No se han definido claramente las políticas de muchos sectores del Poder Ejecutivo, particularmente en el ámbito social, ni la manera en que éstas deben coordinarse con las políticas y decisiones en el nivel provincial y distrital.

Estos problemas generales tienen una serie de manifestaciones en cada una de las dependencias del Poder Ejecutivo, que abarcan aspectos tales como la alta dirección, la carrera de funcionario público, los problemas técnicos inherentes a cada sector o agencia, la coordinación y el control de programas, proyectos y actividades, la información estadística, y los procesos presupuestales. Si bien cada una de estas manifestaciones adquiere características especiales en los diversos sectores y 
agencias, el cuadro $\mathrm{N}^{\circ} 3$ ofrece una visión de conjunto de los problemas del sector público que afectan particularmente al Poder Ejecutivo.

\section{Los riesgos de "más de lo mismo".}

De continuar sin modificaciones significativas el estilo de gestión pública en el Poder Ejecutivo, es probable que las limitaciones se agudicen, se refuercen mutuamente y que socaven los esfuerzos para pasar de la etapa de estabilización a la de reformas estructurales e institucionales.

En primer lugar, de acuerdo al Banco Mundial, la descentralización debe ser uno de los objetivos principales de la reforma del sector público en el Perú, principalmente debido a que la provisión de servicios públicos en el nivel local asegura una mayor eficiencia y genera mayores posibilidades de hacer responsable (accountable) al sector público frente a la población. ${ }^{13}$ Sin embargo, es previsible que el gobierno actual acentúe la centralización y concentración de sus actividades en Lima. Como consecuencia, se mantendrían las asimetrías de información que impiden una asignación de recursos más eficientes en las regiones, se profundizarían los desequilibrios espaciales, y se acentuarían las desigualdades en el acceso a los servicios entre Lima y el resto del país. En estas circunstancias, es poco probable que se generen y mantengan núcleos activos dedicados a identificar proyectos prioritarios y al planeamiento estratégico en las diversas regiones del Perú, por lo cual las decisiones que afectan su desarrollo serían tomadas en Lima por el Presidente y sus principales colaboradores. El riesgo de privilegiar criterios políticos sobre los técnicos aumentaría significativamente, sobre todo en períodos de elecciones. ${ }^{14}$

\footnotetext{
${ }^{13}$ Geoffrey Shepherd, "Reform Issues for Peru's Public Sector", Washington, D.C., Banco Mundial (mimeo), febrero de 1995.

${ }^{14}$ Uno de los rasgos más singulares del estilo de gobierno actual es su tendencia a concentrar y centralizar la administración de los recursos en Lima y el gobiemo central. La distribución del presupuesto para 1997 entre las principales 25 instituciones del sector público indica que existen dos ministerios (economia y finanzas, y presidencia) que concentran el $54 \%$ del total del presupuesto de la República. Si a estos dos ministerios se affaden los Ministerios de Defensa, Interior y Transportes y Comunicaciones, resulta que cinco ministerios manejan el $79 \%$ del total del presupuesto de la República. Esta situación se debe a que, por ejemplo, diferentes prógramas y partidas descentralizadas de sectores como educación, agricultura, energla, salud y saneamiento son administradas por el MEF y por el MIPRE desde Lima. En muchos casos esto implica una asignación de recursos que se basa más en criterios de caja que en fundamentos técnicos $y$, por otro lado, supone la toma de decisiones descentralizables desde Lima muchas veces sin información actualizada y sin tomar en cuenta la diversidad de las diferentes regiones del pais. En el mediano plazo, el proceso de reconstrucción institucional requerirá de un manejo desconcentrado y descentralizado de las politicas y programas.
} 


\section{Cuadro $\mathrm{N}^{\circ} 3$}

\section{Problemas actuales dèl sector público}

\begin{tabular}{|c|c|c|}
\hline Aspecto/Problema & Causas & Consecuencias \\
\hline $\begin{array}{l}\text { Dirección y } \\
\text { Decision }\end{array}$ & $\begin{array}{l}\text { - Divorcio entre el trabajo en linea y los grupos con poder de } \\
\text { decisión. } \\
\text { - Funcionamiento de estructuras paralelas que duplican fun- } \\
\text { ciones y generan inestabilidad. }\end{array}$ & $\begin{array}{l}\text { - No existen condiciones para impie- } \\
\text { mentar un enfoque de gerencia es- } \\
\text { tratégics institucionalizado en el sector } \\
\text { público. }\end{array}$ \\
\hline $\begin{array}{l}\text { Carrera } \\
\text { Pública }\end{array}$ & $\begin{array}{l}\text { - No existe la carrera pública. } \\
\text { - Las administraciones no promueven la formación de } \\
\text { cuadros especializados. } \\
\text { - Existe una estructura salarial que tience una insuficiente } \\
\text { diferenciación como para estimular al persónal especiali- } \\
\text { zado. } \\
\text { Los niveles remunerativos de los profesionales están muy } \\
\text { por debajo del mercado. }\end{array}$ & $\begin{array}{l}\text { - Falta personal con alta calificación en } \\
\text { funciones en linea. } \\
\text { - Organizaciones püblicas no tienen ca- } \\
\text { pacidad para convocar cuadros pro- } \\
\text { fesionales de primer nivel: } \\
\text { - No existe continuidad en los cargos } \\
\text { ejecutivos de las entidades claves de } \\
\text { los Ministerios: }\end{array}$ \\
\hline $\begin{array}{l}\text { Problemas } \\
\text { Técnicos }\end{array}$ & $\begin{array}{l}\text { Debilidad de los cuadros técnicos encargados de la formu- } \\
\text { lación y evaluación de pollticas, programas y proyectos. } \\
\text { Carencia de formuladores de politicas y programas en los } \\
\text { diferentes niveles de la administración pública. } \\
\text { Falta,de continuidad en las acciones determina que exista } \\
\text { una precaria memoria institucional. } \\
\text { No existen-mecanismos para promover la productividad. }\end{array}$ & $\begin{array}{l}\text { Se pierden millones de dólares por } \\
\text { errores relacionados a dlágnósticos } \\
\text { equivocados e incapacidad de } \\
\text { ejecución adecuada de politicas y piro- } \\
\text { gramas. } \\
\text { - Las prioridades de'gasto no están fun- } \\
\text { damentadas desde el punto de vista } \\
\text { téenico. }\end{array}$ \\
\hline $\begin{array}{l}\text { Coordinación } \\
\text { Interna }\end{array}$ & $\begin{array}{l}\text { - Operación limitada de los mecanismos de coordinación y } \\
\text { comunicación. } \\
\text { - Deficiente coordinación inter sectorial que opera en muchos } \\
\text { casos de forma informal, debilitandolas legítimas instancies } \\
\text { de coordinación interministerial. } \\
\text { - Falta de coordinación entre agencias para formular pro- } \\
\text { gramas que tienen objetivos convergentes. } \\
\text { - Exceso de rotación de personal en cargos en los que se } \\
\text { requiere estabilidad para articular y coordinar programas. }\end{array}$ & $\begin{array}{l}\text { - Duplicidados de funciones deterninan } \\
\text { pérdida de valiosos rocursos. } \\
\text { - No es posible el seguimiento consis- } \\
\text { tente de los resultados, de programas } \\
\text { que permiten el logro de objetivos, } \\
\text { compartidos. }\end{array}$ \\
\hline $\begin{array}{l}\text { Problemas de } \\
\text { Control } \\
\text { i. }\end{array}$ & $\begin{array}{l}\text { - Excesivos procedimientos hacen lenta la administración. } \\
\text { - Inapropiado diseño e implementación de la función de la } \\
\text { Contralonia. } \\
\text { - Personal mas preocupado por cumplir procedimientos que } \\
\text { resultados. }\end{array}$ & $\begin{array}{l}\text { - El excesivo control administrativo ex- } \\
\text { istente rigidiza la gestión pública y } \\
\text { disminuye su eficacia ostensible. } \\
\text { mente. }\end{array}$ \\
\hline $\begin{array}{l}\text { Problemas } \\
\text { Estadísticos }\end{array}$ & $\begin{array}{l}\text { - Falua de apoyo para la generación de la estadistica básica en } \\
\text { los diferentes niveles de gobiemö. } \\
\text { - Carencia de estadisticas cinformación actuelizada de carác. } \\
\text { ter ceonómico a nivel regional o local: }\end{array}$ & $\begin{array}{l}\text { - No existen las bases estadisticas para } \\
\text { la toma de decisiones. Por tanto se } \\
\text { gerencia "s ciegas". } \\
\text { - Ej planeamiento se dificulta por la in- } \\
\text { capacidad de realizar proyecciones. }\end{array}$ \\
\hline $\begin{array}{l}\text { Finanzas y } \\
\text { Presupuesto }\end{array}$ & $\begin{array}{l}\text { - Debilidad de sisterias de presupuesto, inversión, personal, } \\
\text { - etc. } \\
\text { - Rigidez en los procedimientos (sistema presupuestal). } \\
\text { - Excesiva centralización fiscal. } \\
\text { - Falla de capacidad para vigilar la calidad del gasto público. } \\
\text { - No hay manera de hacer seguimiento de la calidad del gasto. } \\
\text { - Sisitema de presupuesto con excesiva concentración de } \\
\text { poder de decisión en el Vice-Ministro de Hacienda. } \\
\text { - Presupuesto público subordinado a flujo de caja. }\end{array}$ & $\begin{array}{l}\text { - Falta de capacidad de gasto de inver- } \\
\text { sión. } \\
\text { - Decisiones excesivamente centralizadas } \\
\text { utilizan crilerios poco técnicos para asig- } \\
\text { nación de prioxidades de gastos. } \\
\text { - Enormes dificultades para asignar re- } \\
\text { cursos presupuestales a nuevos pro- } \\
\text { gramas. }\end{array}$ \\
\hline
\end{tabular}


En segundo lugar, al carecer de equipos técnico-políticos de primer nivel encargados de la gestión pública superior, se mantendría el desbalance que existe entre las pocas entidades públicas eficientes y la vasta constelación de organizaciones públicas que operan en condiciones de extrema precariedad técnica y operativa. Las modernizaciones jnstitucionales sólo alcanzarían a aquellas instituciones que logren captar la atención y el apoyo del Presidente de la República (o de sus principales asesores), que tengan apoyo de los organismos internacionales, o que logren un cierto grado de autonomía económica y operativa. ${ }^{15}$ En este sentido, lo más probable es que los problemas de excesiva rotación de gerentes y directores, escasez de profesionales de primer nivel, falta de continuidad en las políticas, inexistencia de sistemas de información, poco control de la agenda, incapacidad de establecer prioridades, interferencia política y excesiva atención a los procedimientos en perjuicio de los resultados, persistan en la gran mayoría de instituciones del Estado.

En tercer lugar, existe el riesgo de que se generalicen los procesos de toma de decisión poco transparentes debido a la falta de apertura en la formulación de políticas, y a lo cerrado y excluyente de los grupos que ejercen el poder político. La escasez de políticas sectoriales claramente definidas, que hayan surgido como resultado de un amplio debate y que sean capaces de generar consensos operativos, hace muy difícil establecer objetivos y metas cuantificables para evaluar el desempeño del sector público. A su vez, esto hace imposible el desarrollo de indicadores de gestión que faciliten la rendición de cuentas. Al no existir las condiciones para una gestión moderna y transparente, cualquier flexibilización en los procedimientos de control podría generar problemas adicionales.

\footnotetext{
${ }^{15}$ En los últimos años ciertas organizaciones públicas han logrado niveles de eficiencia superiores a la mayoria de agencias del gobiemo. Entre ellas es posible mencionar a la SUNA'T y la SUNAD, las organizaciones reguladoras del sector financiero (Conasev, SAFP, SBS, etc.) y otros órganos reguladores como PERUPETRO (contratos petroleros), el Osiptel (telecomunicaciones) y el Indeceopi (libre competencia), asi como FONCODES (lucha con la pobreza). $\iota$ Qué patrones comunes han permitido el surgimiento de estas instituciones? Entre las más importantes destacan apoyo politico al más alto nivel, tener recursos suficientes para mantener un nivel de remuneraciones de su personal a niveles de mèrcado y contar con un marco legal que les permite un grado de autonomia mucho mayor al del resto del sector público. Sin embargo, cabe preguntarse ¿es posible replicar estas experiencias en otros sectores? La regulación del transporte aéreo, de los puertos tras su privatización, la fiscalización de las concesiones de carreteras, el control sobre la biomasa del mar peruano (actualmente a cargo del lmarpe), las regulaciones sobre las rutas interprovinciales y urbanas de pasajeros, el control de concesiones forestales son todas actividades susceptibles de contar con órganos reguladores autónomos financiados con un porcentaje de la facturación de las empresas reguladas. El modelo Osiptel, órgano regulador de las telecomunicaciones, puede ser un camino a seguir. Sin embargo, la creación de órganos reguladores autónomos y especializados tendrá efectos positivos en la capacidad de regulación del Estado siempre y cuando estos organismos cuenten con un marco legal adecuado, un alto grado de autonomla financiera y operativa, $y$ con un fuerte apoyo político.
} 
En cuarto lugar, debido a las limitaciones en la formulación de políticas los procesos de privatización, y en particular las privatizaciones de servicios públicos, corren el riesgo de desvincularse de las políticas y de los objetivos de mediano y largo plazo para el respectivo sector y para el Poder Ejecutivo en su conjunto. Más aón, sin políticas y objetivos claros; es posible que predominen sólo los criterios referentes a los ingresos por la venta de empresas estatales, en desmedro de las consideraciones de calidad, oportunidad y cobertura en la provisión de servicios públicos, y de los procedimientos y costos de la regulación.

En quinto lugar, la ausencia de esfuerzos de concertación con los diversos grupos de la ciudadanía, incluyendo a la oposición política, en temas que requieren un horizonte temporal de largo plazo puede generar inestabilidad y afectar el desempeño de las diversas entidades del Poder Ejecutivo. Esto es particularmente importante en lo referente a la reforma del Poder Ejecutivo, la creación de un servicio civil, la modificación de procedimientos presupuestales y de control, y muchas otras iniciativas que requieren de más de dos o tres períodos de gobierno para rendir frutos. La experiencia de muchos intentos de reforma durante los últimos decenios demuestra claramente que sin un amplio consenso alrededor de un núcleo de reformas institucionales, no es posible modernizar e introducir reformas en el aparato estatal.

Por último, es previsible que se mantengan las deficiencias existentes en los principales mecanismos para igualar oportunidades (programas de salud, educación, empleo, capacitación laboral). Esto se explica tanto por la prioridad otorgada a los programas temporales de lucha contra la pobreza y compensación social, como por la carencia e ineficiencia de programas de mediano y largo plazo destinados a reforzar los ministerios, municipalidades y otras agencias encargadas de los programas sociales. Si bien el gobierno ha logrado notables éxitos en contener el proceso de deterioro en la calidad de vida, y ha disminuido el ritmo de aumento de la pobreza absoluta, las reformas económicas han tenido como consecuencia una mayor desigualdad del ingreso y del consumo, y una creciente brecha entre los peruanos ricos y pobres. ${ }^{16}$ Este aumento

\footnotetext{
${ }^{16}$ La ineficacia de los mecanismos para generar empleo adecuadamente remunerado y suministrar servicios sociales con un minimo de calidad están ampliando la brecha entre ricos y pobres. Es decir, está aumentando la pobreza relativa expresada en términos de las diferencias en ingresos entre las familias de ingresos altos (nivel socio-económico A) y medios (nivel B) versus los ingresos de las familias de ingresos bajos (nivel C) y muy bajos (nivel D). Según encuestas de Apoyo S.A., los ingresos mensuales de las familias del nivel A pasaron de US\$2.823 a US\$4.040 entre 1991 y 1996. Es decir, tuvieron un aumento de sus ingresos equivalente a US\$1.113. Por su parte el Nivel B registró en el mismo perfodo un aumento de sus ingresos mensuales equivalente a US\$369. En contraste el aumento de los ingresos mensuales de las familias de los niveles Cy D sólo ascendió a US\$92 y US\$197, respectivamente.
} 
en los índices de desigualdad, que se añade al deterioro que produjo la crisis económica de fines del decenio de los ochenta, podría tener como consecuencia un incremento en los índices de criminalidad y de violencia política.

\section{Pautas para la reforma del Poder Ejecutivo.}

Para avanzar hacia una estructura del Estado y una reforma del Poder Ejecutivo capaces de responder a los desafios de la transición a un nuevo siglo, es preciso adoptar una perspectiva de largo plazo, lograr acuerdos sobre la visión de futuro y definir un marco de políticas globales y sectoriales para orientar nuestro proceso de desarrollo. A su vez, esto exíge avances en la dirección de la gobernabilidad democrática, profundizando los procesos de modernización productiva, democratización social y legitimación de las instituciones, particularmente las instituciones estatales.

\section{Estrategia de desarrollo y reforma del Estado.}

El diseño de una estrategia integral de desarrollo, que debe tomar en cuenta una noción ampliamente compartida del bien común y del interés nacional, es aún una tarea pendiente en el Perú de hoy. Luego de concluido el diagnóstico de los problemas de gobernabilidad democrática que enfrenta nuestro país, el trabajo actual de Agenda: Perú está orientado hacia el diseño participativo de uṇa visión compartida del futuro y de una estrategia de desarrollo a largo plazo. Como resultado de estos trabajos será posible contar con lineamientos para una reforma del Estado y del Poder Ejecutivo que incluirá, por ejemplo, una propuesta detallada sobre la estructura de los ministerios, entidades públicas descentralizadas y agencias del gobierno central. ${ }^{3}$

Sin embargo, es posible adelantar algunas ideas sobre la estrategia de desarroilo que fundamentan los trabajos de Agenda: Perú sobre reforma

\footnotetext{
${ }^{17}$ El trabajo sobre estrategias de desarrollo de Agenda: Perí se basa en: (i) la información recabada y procesada durante 1993-1995, que llevó a la publicación del documento Democracia y Buen Gobierno (cuya segunda edición se presentó en septiembre de 1996); (ii) en varios trabajos que viene realizando Agenda: Peri sobre reforma del Estado, identidad cultural y conflicto, el orden global fracturado, y otros temas similares; $y$ (iii) en el trabajo realizado entre 1993 y 1996 en GRADE sobre estrategias de desarrollo por un numeroso equipo de investigadores y consultores dirigidos por uno de los co-directores de Agenda: Perí. Todo este material debe ser reprocesado, integrado y actualizado para elaborar una primera versión de la estrategia integral de desarrollo, que será revisada luego del proceso de consultas.
} 
del Estado y del Poder Ejecutivo. ${ }^{18}$ Entre las líneas estratégicas identificadas es posible mencionar:

- El proceso de modernización productiva, que debe llevar hacia un sistema económico moderno y competitivo, hacia la extensión del mercado como mecanismo para asignar recursos, y hacia un proceso de acumulación viable y sostenible en el tiempo. Además de una política macroeconómica sensata, esto exige una serie de políticas sectoriales dirigidas hacia reducir la exclusión económica.

- El proceso de democratización social, que debe llevar hacia una reducción de las desigualdades extremas (en sus dimensiones de pobreza, geografía, género, edad, raza y cultura), hacia la provisión de servicios sociales básicos a toda la población, y hacia el respeto irrestricto a los derechos humanos. El resultado será una sociedad civil vigorosa y activa, y un Perú más integrado socialmente. Esto exige una serie de políticas y medidas orientadas hacia reducir la pobreza endémica y la pobreza crónica que afectan a un elevado porcentaje de la población.

- El proceso de legitimación de las instituciones del Estado, que debe articular una concepción compartida del bien común y del interés nacional, y que debe llevar hacia un conjunto de instituciones estatales eficientes y representativas, capaces de superar la exclusión política, y con las cuales se pueda identificar la ciudadanía. Además de centrar la atención en las instituciones del Estado, el proceso de legitimación debe explorar las vinculaciones de estas instituciones con el sector privado y las organizaciones de la sociedad civil, articulándolas para aprovechar complementariedades. Este proceso también involucra una redefinición de las relaciones entre las Fuerzas Armadas y la sociedad civil, con el fin de garantizar las condiciones para la gobernabilidad democrática.

- Un conjunto de procesos dirigidos hacia reducir la exclusión de las generaciones futuras, que abarcan: (i) la preservación del medio ambiente, la reducción de la contaminación y el uso sustentable de los recursos naturales; (ii) la creación de una capacidad científica y tecnológica, que permita absorber tecnologías importadas, rescatar y mejorar las tecnologías tradicionales, y desarrollar nuevas tecnologías para apoyar los procesos de modernización productiva y democratización social; y (iii) medidas para reducir la violencia en todas sus

\footnotetext{
${ }^{18}$ Francisco Sagasti, "Pobreza, Exclusión y Estrategias de Desarrollo", (Lima: Agenda: Peru, agosto de 1996), (versión preliminar).
} 
formas, con el fin de crear las condiciones para recomponer el tejido social y avanzar hacia una cultura de la paz que estimule la acción solidaria.

- Un proceso de integración cultural orientado hacia forjar una concepción compartida de la identidad nacional, que debe promover el respeto y la tolerancia a la diversidad, y revalorizar la enorme variedad de manifestaciones culturales que caracterizan al Perú. Este proceso contribuirá significativamente a promover la autoestima y la confianza en el futuro.

Estos lineamientos para el diseño de una estrategia de desarrollo establecen pautäs para la reforma del Estado, así como de sus vinculaciones con las organizaciones de la sociedad civil y el sector privado. Todo esto nos lleva hacia una redefinición del papel-del Estado en el desarrollo peruano, que debe tomar en cuenta no sólo las condiciones internas del Perú, sino también las exigencias de urr orden global fracturado en el ámbito internacional que impone limitaciones a la acción estatal, pero que también genera oportunidades que pueden y deben ser aprovechadas.

\section{Las funciones generales del Estado.}

De las consideraciones sobre estrategia de desarrollo es posible deducir las funciones que competen al Estado. Partiendo de la idea que el Estado es responsable por definir la noción del bien común y el contenido de los intereses nacionales, es posible delinear las siguientés funciones estatales:

- Garantizar la seguridad externa e interna.

- Administrar la provisión de justicia.

- Normar, vigilar y defender la estabilidad de las reglas de juego para los agentes económicos, incluyendo a los consumidöres y productores de bienes y servicios.

- Estimular y promover la competitividad de las empresas productoras de bienes y servicios; así como su adecuáda insercion en la división internacional del trabajo (incluýendo la provisión de servicios técnicos y el apoyo a la innovación tecnológica).

- Normar, vigilar y defender la estabilidad de lás reglas de juegó para la participación y la representación política, (incluyendo la defensa de la libertad de expresión, el funcionamiento adecuado del sistema electoral, y la ampliación de mecanismos para la participación ciudadana). 
- Promover la equidad, la integración y la justicia social, (incluyendo la ampliación de oportunidades de empleo, la provisión de servicios sociales básicos, ia igualdad de oportunidades y los mecanismos para redistribuir el ingreso).

- Promover la integración cultural y el respeto a la diversidad, (incluyendo la transmisión de valores ciudadanos y la formación de una conciencia común entre los peruanọs).

- Normar y realizar el planeamiento estratégico de largo plazo para el uso de los recursos naturales (medio ambiente), la ocupación del territorio y promoción del desarrollo equilibrado de las regiones.

El adecuado cumplimiento de estas funciones genéricas requiere de una reforma integral del Estado que involucra al Poder Ejecutivo, y a los poderes Judicial, Electoral y Legislativo, y que trasciende los procesos de modernización que generalmente se asocian con la estabilización y las reformas de política económica (Véase los cuadros $\mathrm{N}^{\circ} 1$ y 2).

\section{Criterios y objetivos para la reestructuración del Poder Ejecutivo.}

En el proceso de reforma del Poder Ejecutivo es necesario aceptar, en primer lugar, que no existe "la reforma del aparato del Estado" como hecho aislado que puede ejecutarse "de una sola vez y para siempre". Es imposible poner en práctica en forma simultánea y de manera inmediata los cambios requeridos en el Poder Ejecutivo para adecuarlos a la estrategia de desarrollo. Más aún, en un contexto internacional cambiante, es necesario que el aparato del Estado sea capaz de adaptarse continuamente a nuevas exigencias, lo que implica adoptar una perspectiva de mediano y largo plazo para el proceso de reformas.

Por otra parte, la reforma del Estado es indesligable del diseño, ejecución y evaluación de políticas. La reestructuración del Poder Ejecutivo debe acompañar a las reformas de política económica y social, de tal forma de vincular su estructura organizativa y sus procedimientos administrativos al logro de objetivos y metas. Asimismo, la reestructuración del Poder Ejecutivo requiere de un conocimiento detallado de la situación actual y de las posibilidades de mejora del sector público, con el fin de diseñar medidas e instrumentos de reforma, así como establecer prioridades y definir una secuencia para su ejecución. Sin embargo, es necesario distinguir claramente entre el contenido y los objetivos de las políticas por un lado, y los instrumentos para ponerlas en práctica, tales como la privatización, la desregulación, la descentralización y la participación de los beneficiarios y usuarios. 
En todo este proceso de reestructuración del Poder Ejecutivo, es preciso reorientar la acción de las organizaciones gubernamentales hacia la obtención de resultados, poniendo un énfasis relativamente menor en los cambios en los marcos legales. Las sucesivas reformas y modernizaciones del Estado peruano han privilegiado el diseño y la modificación de leyes y reglamentos, e implícitamente han supuesto que promulgar-o derogar- normas legales es suficiente para mejorar el desempeño del sector público. Esto no disminuye la importancia de los marcos legales, pero busca ubicarlos en su lugar correcto. (Véase el cuadro $N^{\circ} 4$ ).

A partir de estos criterios es posible definir un conjunto de objetivos para la reforma del Poder Ejecutivo, entre los cuales se encuentran:

- Adecuar las estructuras organizativas del sector público a las necesidades de la estrategia de desarrollo.

- Aumentar la eficiencia y la eficacia del proceso de toma de decisiones, fortaleciendo la capacidad de formular y ejecutar políticas. Esto requiere prestar especial atención al nivel de la gerencia pública superior.

- Modificar la estructura y los procedimientos operativos de las diversas entidades del Poder Ejecutivo, particularmente en el ámbito de las políticas sociales, en función de una descentralización política y administrativa.

- Mejorar los procedimientos de coordinación entre las diversas instituciones y organizäciones del Poder Ejecutivo, reducir la duplicidad y el traslado de funciones, y eliminar las rigideces en los procedimientos de control que traban la gestión en el sector público.

- Crear instancias para el diálogo, promover consensos operativos y ampliar la participación de los diversos actores sociales, con el fin de involucrar activamente a los ciudadanos en las tareas del desarrollo nacional.

Pautas para la reestructuración del Poder Ejecutivo.

Estos criterios y objetivos permiten definir una serie de pautas que permitirán elaborar propuestas detalladas para la reorganización del Poder Ejecutivo. Estas pautas se pueden resumir en los siguientes términos:

- Conformar gabinetes de asesores especializados en gestión pública superior para apoyar al Presidente de la República y al Presidente del Consejo de Ministros. Estos gabinetes deben fortalecer la capacidad de diseño de políticas públicas tomando en cuenta los acuerdos 
políticos de mediano y largo plazo. Pasada la etapa de emergencia nacional y de estabilización de la economía, las tareas del desarrollo peruano en el futuro requieren de una perspectiva de mediano y largo plazo, y de un proceso de creación y refuerzo de instituciones, que con seguridad abarcarán varios gobiernos. Por esta razón el Poder Ejecutivo requiere de una visión estratégica de largo alcance que incorpore aspectos políticos y técnicos, así como mecanismos de coordinación que abarquen a los otros poderes del Estado.

- Configurar un Consejo de Ministros más pequeño y operativo, con un mejor equilibrio en la distribución de funciones y responsabilidades, y que sea una institución más ágil y operativa, con mayor control sobre su propia agenda y centrado en la evaluación de los grandes temas de las principales políticas de desarrollo nacional.

- Reagrupar los ministerios y agencias en los diversos sectores de Poder Ejecutivo, con el fin de lograr una mayor coherencia en la formulación y ejecución de políticas.

- Trasladar y delegar atribuciones y funciones administrativas internas, incluyendo aquellas referidas al gasto público y la ejecución presupuestal, de los ministros a los secretarios de Estado, con el fin de que los ministros se concentren en las tareas de çonducción de las políticas sectoriales a su cargo, y de su vinculación con las políticas generales del gobierno.

- Conformar gabinetes con personal altamente capacitado, pequeños y flexibles, para asesorar cada uno de los ministros en asuntos políticos, formulación de políticas planeamiento estratégico, innovación tecnológica y análisis de problemas complejos.

- Diferenciar las funciones al interior de cada ministerio, dividiéndolas entre aquellas instancias que definen las orientaciones generales y el planeamiento estratégico (secretarías de Estado), las entidades que definen la normatividad de cada sector (direcciones generales), las entidades encargadas de aplicar las normas (agencias reguladoras) y las entidades encargadas de la provisión de bienes y servicios (instituciones públicas descentralizadas y empresas públicas).

- Descentralizar los programas de salud, educación, saneamiento ambiental, protección del medio ambiente y provisión de infraestructura, entre otros, para articular mejor la acción del gobierno central, particularmente en el nivel de la gestión pública superior, y las instancias regionales y locales. La descentralización implica una transferencia progresiva y gradual de responsabilidades y recursos, y una eva- 
luación permanente el desempeño de los programas descentralizados en términos de calidad, cobertura, oportunidad y costos involucrados. - Diseñar y poner en práctica sistemas de información para la gestión pública que permitan disponer de la información adecuada y de los datos pertinentes para el diseño, ejecución, seguimiento y evaluación de los programas y proyectos del sector público.

Estas pautas permitirían contar con un Poder Ejecutivo más transparente, eficaz, eficiente y orientado hacia resultados, capaz de poner en práctica una estrategia de desarrollo con perspectiva y visión de largo plazo. Permitirían, además, contar con cuadros profesionales técnicos y políticos, agrupados en el nivel de la gestión pública superior, con pleno conocimiento de la realidad de las diversas entidades del Poder Ejecutivo y con una comprensión clara de los objetivos de la política gubernamental. Para complementar estas pautas, el cuadro No5 presenta las características de lo que sería una agencia gubernamental eficiente.

\section{Cuadro $\mathrm{N}^{\circ} 4$ \\ Características de los marcos legales eficientes}

- Deben ofrecer estabilidad jurídica, sobre todo en aquellas instituciones que de acuerdo a su función están en condiciones de promover la participación de la inversión privada.

- Deben estar estructurados de modo tal de adecuarse a los cambios del entorno y de la tecnología.

- Deben evitar duplicidad de funciones entre organizaciones públicas.

- Deben promover la transparencia en el proceso de toma de decisiones.

- Deben establecer obligaciones de la organización pública de entregar la información necesaria para que el usuario -o beneficiario- puede elegir libremente los servicios.

- Deben prever mecanismos claros, viables y expeditivos para resolver controversias.

- Deben considerar todos los aspectos centrales referidos a la actividad de la organización y ser consistentes con la normatividad del sector en el que la organización se desenvuelve.

- Deben considerar la participación de los usuarios de los servicios.

- Deben ser fáciles de entender, supervisar e implementar.

- Deben establecer un código de ética entre sus funcionarios de modo de evitar conflictos de interés. 


\section{Cuadro $\mathrm{N}^{\circ} 5$}

\section{Características de una agencia gubernamental eficiente}

\section{ESTRUCTURA}

- Visión de futuro: El directorio y sus principales ejecutivos deben compartir una visión de futuro del sector en el que se desenvuelven y de su papel en el marco de las politicas generales del gobierno.

- Presúpliesto: Debe evaluarse la manera de que los presupuestos de las organizaciones sean proporcionales a su responsabilidad, jurisdicción y poder económico de las organizaciones privadas afectadas por su actividad.

- Autonomia: La reforma debe procurar que las organizaciones, en la medida de lo posible, cuenten con autonomía administrativa, técnica, económica, financiera y funcional.

- Flexibilidad: Cada organización deberia convertirse en un ente pequeño, tecnificado y flexible que promueva la eficiencia económica de la actividad a la que se relaciona al más bajo costo sin perder la capacidad de cumplir los objetivos inherentes a su mision. Las organizaciones deben estar estructuradas de modo de ser los suficientemente flexibles como para responder adecuadamente a los cambios tecnológicos y estructurales que se produzcan er el sector.

\section{PERSONAL}

- Profesionalismo: El plantel de cada organización debe estar compuesto por profesionales de primer nivel que reciban una remuneración equivalente a la existente en el sector privado relacionado a la institución al mismo nivel de responsabilidad o, por lo menos, una remuneración competitiva de mercado.

- Meritocracia y capacitación: Las organizaciones debe evaluar imparcialmente el desempeño de cada trabajador y capacitarlo continuamente.

- Manejo de la agenda: Los directivos de las organizaciones deben lograr un manejo adecuado de sus agendas sobre la base de un planeamiento estratégico.

- Límites de su intervención: Los directivos de las organizaciones deben comprender a cabalidad cuáles son los limites de su intervención.

- Desarrollo del personal: Cada organización debe asumir que su capital humano es el centro de gravedad de la institución. Esto implica comprender que de su capacidad de diagnóstico y ejecución dependerá, fundamentalmente, el éxito en relaciỏn al cumplimiento de su misión. Se debe promover una atmósfera de trabajo que permita al desarrollo personal de todos los trabajadores y refuerce la iniciativa individual para que cada uno de ellos alcance su máximo potencial.

- Creatividad: Las organizaciones deben promover la creatividad de su miembros, buscando los resultados a través del mantenimiento de una estructura de toma de decisiones flexibles que evite la burocratización.

- Confianza: Las relaciones entre los directivos y el personal se establece sobre la base de la confianza como mecanismo para obtener el máximo de compromiso del personal con los fines de la institución.

\section{PROCEDIMIENTOS}

- Diálogo: Los directiyos de la organización deben tener capacidad de mantener un diálogo fluido con la sociedad.

- Trabajo en equipo y multidisciplinariedad: Las organizaciones deben promover el trabajo en equipo que permita la construcción de marcos analiticos y enfoques interdisciplinarios para encarar los desafios que enfrenta la institución.

- Imparcialidad: La organización debe tomar decisiones a partir de un análisis imparcial que considere los puntos de vista de todos los agentes involucrados y evalúe su impacto sobre los mismos.

- Transparencia: La organización debe mostrar un comportamiento consistente, transparente y predecible a fin de reducir el riesgo y la incertidumbre.

- Rendición de cuentas: Cada organización debe desarrollar sus mecanismos de rendición de cuentas. 